Fig. 22. Ganglienzellen. Korntinction mit Hämatoxylin und Jodgrün. E ngel bert and Hensold Syst. V. Imm. Oc. 2.

$$
\left.\begin{array}{l}
\mathrm{n}=\text { unipolare } \\
\mathrm{r}=\text { runde } \\
\mathrm{b}=\text { bipolare }
\end{array}\right\} \text { Zellen. }
$$

Fig. 23. Theil eines mit dem Mikrotom aus dem Scolex entnommenen Querschnittes. Inge 1 b e $\mathrm{t}$ and $\mathrm{H}$ e nso $1 \mathrm{~d}$ t. Syst. 5. Imm. Oc. 2. $\mathrm{gh}=$ Ganglionhülle.

Die ïbrige Bezeichnung wie vorher.

Fig. 24. Brïckencommissur. Engelbert ind $H$ ens ol d Syst. 5. Imm. Oc. 2.

Fig. 25. Quersehnitt durch den hinteren Alschnitt einer Proglottide aus der Mitto der Strobila. En gel bert und Hensoldt Syst. 4. Oc. 2. sn $=$ Seitennerv.

Die ubrige Bezeichunug wie vorher.

Fig. 26. Längsschnitt durch den Seitennerven.

$\mathrm{g}=$ Ganglienzelle.

na $=$ ausgetretener Nervenast.

(Aus dem anatomischen Institute zu Breslau.)

\title{
Die Plattenmodellirmethode
}

von

Dr. G. Born.

Als ich im Jahr 1876 die Entwicklung der Nasenhöhle und des Thränennasengangs der Amphibien an Schnittserien studirte, machte mir die Schwierigreit, die complicirten Schnittfiguren der Organe der Ethmoidalgegend im Kopfe wieder zu einem richtigen, plastischen Bilde zusammenzusetzen so sehr zu schaffen, dass ich auf die Idee kam, diese Reconstruction, die die Pbantasie nach Maass und Zahl nur sehr unvollkommen zu leisten vermochte, objectiv mittelst Zusammenlegen in Fläche und Dicke gleichmässig vergrösserter Wachsplatten herzustellen. 
Die nothwendigste Voraussetzung fïr einen solchen Versuch, nämlich eine annähernd constante Dicke der serienmässig angefertigten Schnitte, war damals für mich schon gegeben, da ich gelernt hatte das Long'sche Schlittenmikrotom und die Kleinenberg'sche Spermacetieinschmelzung für meine Zwecke zuverwenden; eine Schwierigkeit blieb noch die Herstelling planparaleler Wachsplatten von bestimmter gleichmässiger Dicke. Doch auch die Lösung dieser Aufgabe gelang, indem ich die aus dem specifischen Gewichte und dem Volumen der gewiinsebten Wachsplatte berechnete Wachsmenge im geschmolzenen Zustande auf heisses Wasser in ein viereckiges Blechgefäss gooss, dessen Wände denselben Flächenraum, wie die Wachsplatte, umspannten.

Nach der hiererst 'kurz angedenteten Methode habe ich bei einer ganzen Reibe entwicklungsgeschichtlicher Arbeiten plastische Modelle der in Schnittserien zerlegten Objecte angefertigt und einzelne davon auch auf den Tafeln meiner Arbeiten abbilden lassen 1).

Mein Verfahren hat dann in einer von $G$. Swirski in Dorpat gearbeiteten Dissertation $\left.{ }^{2}\right)$ Nachahmung gefunden; noch mohr aber in einer ganzen Reile von Arbeiten meines Freundes und Collegen Ph. St ij $\mathrm{r}$ in Wirzburg ${ }^{3}$ ), den grosse technische Geschicklichkeit und Feinheit ganz besonders zu derartigen Leistungen befühigen. Stijhr hat dann in nenester Zeit von Herrn Ziegler in Freiburg i. Br. Nachbildungen seiner Modelle des Primordialschädels der

1) Die erste Beschreibung des Verfahrens ist zu lesen: Morphol. Jahrb. Bd. II. 1876 ,Ueber die Nasenhöhlen und cLen Thränennasengang der Amphibien" p. 578-80. Abbitdungen von Modellen finden sich in "Die Nasenhöhlen und der Thränemasengang der amioten Wirbelthiere. I. Morphol. Jahrb. Bd. V. - dito III. Morphol. Jahrb. Bd. VIII. - Ueber die Derivate der embryonalen Schlundbogen und Schlundspalten bei Säugethieren, Archiv f. mikroskop. Anat. Bd. XXII, Heft 2.

2) Untersuchungen über die Entwicklung des Schultergürtels und des Skelets der Brustflosse des Hechts. Dorpat 1880.

3) Ph. Stöhr, "Zur Entwicklungsgeschichte des Urodelenschädels", Zeitschr. f. wissensch. Zool. Bd. XXXIII. - „Zur Entwicklungsgeschichte des Anurenschädels:, Zeitschr. f. wissenschaftl. Zool. Bd. XXXVI. - „Zur Entwicklungsgeschichte des Kopfskelets der Teleostier". Festschr. d. medic. Facultät zu Würrburg. 1882. Bd. II. - Prospectus über die von Dr. Ad. Ziegler in Freiburg i. B, angefertigten Unterrichtsmodelle, die Bildung des Primordialschädels erlänternd. 1882. 
Amphibien und Fische herstellen und in den Handel bringen lassen. Allerjuingst hat Herr Dr. Uskow, den ich hier in Breslan zuerst in die Entwicklungsgeschichte und in die entwicklungsgeschichtliche Technik eingefuhrt habe, die Methode benutzt, wie aus folgenden Ang’aben seiner Arbeit „Ueber die Entwicklung des Zwerchfelles, des Pericardiums und des Coeloms" aus dem anatomischen Institute $\% u$ Strassburg. ${ }^{1}$ ) hervorgeht: ,Um eine klare Vorstellung von der Configuration der besprochenen Gegend zu erhalten, haben wir nach mikroskopischen Präparaten ein Wachsmodell hergestellt".

Däu die Anm.: „Mit Hülfe der Camera lacida wurden (ähnlich wie es, glaube ich, zuerst von $B$ ö $\mathrm{n}$ vorgeschlagen ist) Zeichnungen auf Wachsplatten gemacht, die Spalten und Lüicken ausgeschnitten, die äusseren Contouren beschnitten, die den successiven Schnitten entsprechenden Wachsplatten in derselben Reihenfolge aufeinander gelegt und zusammengeklebt. Auf diese Weise wurdo der grösste Theil der unten zu beschreibenden Entwicklungsstadien getreu nacli den Präparaten reproducirt."

Es ist nun, wie auch $\mathrm{His}^{2}$ ) wieder und wieder hervorhebt, bei entwicklungsgeschichtlichen Arbeiten absolut nothwendig; sich "tiber die blosse Schnittbetrachtung za einer plastischen Synthese des zerlegten Gebildes zu erheben“ und ,die zahlreichen Flächenbilder, welche die einzelnen Schnitte gewähren, wieder umzusetzen in einfachere Anschaunngen körperlicher Art." $\mathrm{His}$ selbst erreicht dieses Ziel bekanntlich dadurch, dass er jeden einzelnen Querschnitt in bestimmter Vergrösserung zeichnet und nach den Maassen dieser Figuren und nach der bekannten Sehnittdicke mit Hïlfe der vor dem Schneiden vom unversehrten Object abgenommenen Bilder Frontal- und Sagittal-Schnitte und Ansichten nach leicht verständlichen Regeln construirt und aufzeichnet. Nach diesen auf.gleiches Maass reducirten verschiedenen Ansichten des Objects wird dann eine freie Nachbildung in Wachs oder Thon ausgefiihrt. So sind die Orginale der fitr jeden Unterricht in der Entwicklungsgeschichte unentbehrlichen Modelle von $\mathrm{H}$ is iber dic Entwicklung des Hilhnchens, die Ziegler in Freiburg liefert, entstanden; ich brauche daher kein Wort dartiber zu verlieren, dass auf diesem Wege Ausgezeichnetes erreichbar ist. Nur ist die fiur diesen Weg unerlässliche, hohe kinstlerische Begabung und Fertigkeit nicht Jedermanns Sache, es scheint mir daher, dass meine

1) Arch. f. mikr. Anat. Bd. XXII. H. 1. p. 157.

2) Anatomie menschlicher Embryonen 1. Leipzig 1880. p. 7. 
Methode, die ohne jeden Anspruch an besondere Fertigkeiten bei rein mechanischer Handhabung richtige plastische Reconstructionen liefert, etwas bäufiger Anwendung verdient, als ihr bisher zu Theil geworden ist. Da ich seit der ersten Veröffentlichung der Methode in der Finleitung einer Specialarbeit') dieselbe immer weiter ausgebildet habe, die Schnittserientechnik selbst aber inzwischen mannichfache, der Ausführung der Modelle guinstige Verbesserungen erfahren hat, scheint es mir an der Zeit, das Verfahren, wie ich es jetzt tube, mit allen nothwendigen Vorbereitungen einmal in extenso darzustellen; vielleicht erreiche ich danit, dass es häufiger in Gebrauch kommt; - die Nitzlichkeit desselben wird sich dann ohne Weiteres ergeben: Ich werde im Folgenden auch auf die einleitenden Manipulationen des Einschmelzens, Schneidens, Auflegens u. s. f. wie ich dieselben als zweckmässig für die Erfordernisse des Modellirens ansgearbeitet habe, etwas genaner eingehen, ohne damit ausschliessen zu wollen, dass dasselbe Ziel anch auf anderem Wege, als auf den von mir angegebenen, erreichbar ist.

\section{Vorbereitende Manipulationen.}

Fir viele Objecte erweist es sich als zweckmässig, bevor man dieselben in Schnitte zerlegt, orst eine in bestimmter Vergriosserung gehaltene Umrisszeichnung anfzunehmen, die dann beim Modelliren als Controlle mannigfach bentitzt werden kann. In meiner letzien Arbeit dienten die Profilzeichnnngen der Köpfe der Embryonen dazu, die Verschiebung in dorsoventraler Richtung, die die Wachsplatten beim Aufeinanderlegen $z \mathfrak{u}$ erleiden hatten, abzumessen: $\mathrm{H}$ i s ${ }^{2}$ ) beniitzt zum Zeichnen eine besondere Apparatenzusammenstellung; da ich eine solche nicht besitze, muss ich mich mit dem Mikroscope behelfen; fiur stärkere Vergrösserungen, 60, 80, 100 fach, wie sie beim Modelliren häufig gebrancht werden, reicht die Combination von $\mathrm{H}$ is tibrigens sowieso nicht ans. Mit einer Reihe Objectiven von Schiek 00 an anfwärts, einigen verschieden hohen Unterlagen und der Oberhäuser'schen Camera lucida oder dem

1) Siehe Anm. 1 p. 585.

2) Anatomie menschlicher Embryonen I. Leipzig 1880. p. 8 u. folg. 
Bruel'schen Zeichenapparate lassen sich die häufigsten Vergrösserungen leicht zusammenstellen, namentlich wenn man den Tubus des Mikroskops je nach Bediurfniss verschieden weit zusammenschiebt oder auszieht. Ich messe mir die Grösse des Auszugs des Tubus, die für irgend eine Vergrösserung erforcterlich ist, ein für allemal ab, schneide einen etwa 3-5 mm breiten Streifen Blech von entsprechender Länge ab und klemme denselben zwischen den vorspringenden Rand der unteren Tubushälfte und den oberen Ring der oberen Tubushälfte fest ein; damit ist die erforderliche Grösse des Auszugs dauernd fixirt. Der Blechstreifen kann jeden Angenblick entfernt und mit Leichtigkeit durch einen neuen ersetzt werden. Will man das Bild des ganzen Objectes als Controlle bein Modelliren benutzen, so ist zweckmässig dasselbe auf dieselbe Vergrösserung umzuzeichnen, in der man modellirt, und die Schnittgrenzen, wie dies $H$ is vorschreibt, auf dem Bilde in parallelen Linien aufrutragen. $H$ is sucht die Schnittrichtung nachträglich zu bestimmen, incem er besonders markante Punkte des Oberflächenbildes in den Schnitten aufsucht und nach der Zahl der Schnitte, die zwischen zwei solehe fallen, und nach der bekannten Schnittdicke die Lage der Linien construirt. Ich schneide immer in einer zu irgend einer Axe des Objects von vorn herein fixirten Richtung; - wie dies geschieht, ist weiter unten nachzulesen ich kann die Schnittlinien daher auch ohne Weiteres in das Oberflächenbild eintragen. Wählt man die Plattendicke beim Modelliren, was sich mitunter empfiehlt, nicht in Bruchtheilen von Millimetern, sondern in ganzen Millimetern, so kaun man eine Durchzeichnung des Umrisses des Objects auf Oelpapier auf käufliches Millimẹterpapier in der bestimmten Richtung aufkleben und erspart sich so das immerhin miihsame Ausmessen und Aufzeichnen der Schnittgrenzen.

Weun man Objecte in Schnittserien von gleichmiässiger bestimmter Dicke zerlegen will, mitssen dieselben natiurlich vorher uweckentsprechend gehärtet und gefärbt sein. Ich brauche hier auf die itblichen Methoden nicht weiter einzugehen, erwähnen will ich nur, dass bekanntlich zum Stickfärben keinesweg's alle Methoden, die fuir das Schnittfärben gutes leisten, anwendbar sind.

Es möchte sich empfehlen anstatt des sohlechten Ausdrucks „Im Ganzen Färben" die Bezeichnung "Stückfärben und Stïckfärbung“ als Gegensatz zu "Schnittfärben und Schnittfärbung" festzuhalten. Als brauchbare Combinationen kemne ich aus eigener Erfahrung: Chromsäurebehandlung, Boraxcarmin oder 
saures Hämatoxylin nach Ehrlich; - Pikrinsïurebehandlung, gewöhnliche ammoniak. starke Carminlösung oder classelbe Hämatoxylin; - Müller'sche Flüssigkeit - oder Alkoholbehandlung, Cochenilleabknchung; - Salpetersäurebehandlung, alkalische Methylvioletlösung $u$. đergl. mehr.

Zum Einbetten benutze ich ausschliesslich Paraffingemische oder die mit etwas Wachs versetzte Wallrathrizinusölmasse von Kleinenberg, ohne dass ich damit leugnen will, dass man mit Eiweissoder Celloidineinschluss nicht anch zum Ziele kommen könnte. Ja es scheint fur den ersten Augenblick, als ob die letztgenamnten Methoden den grossen Vorzug besässen, dass die Schnitttheile von der Einschlussmasse, die mit auf den Objecttriger kommt, festgehalten viel weniger leicht der Gefahr einer Verzerrung oder Verschiebung ausgesetzt sind; aber einerseits leistet eine gute Paraffin- oder Wallrathmasse, wenn man mit trockenem Messer schneidet dasselbe; der Schnitt sammt dem umgebenden Plattchen der Masse wird auf den Objectträger aufgelegt und auf diesem sogleich, wie unten näher auszufuhlen, datternd fixirt, - andererseits gewährt meine Einsschlussmasse, wenn man ihre Plasticitait, wie ich vorschlage, zweckmässig ausnützt, eine Leichtigkeit und Sicherheit in der Orientirung des zu schneidenden Objects, wie dies kein anderes Verfahren zu gewihluren vermag. Vor dem Einschmelzen lege ich die Stiicke je nach der Grösse längere oder kìirzere Zeit in Bergamottöl ein, bis sie von demselben vollkommen durchdrungen sind, - neuerdings wird dafür auch Chloroform empfohlen, - nud bringe sie daun in die geschmolzene Masse, die auf einem Wasserbade oder noch besser in einem mit Wasser heitzbaren Trockensehränkchen grade flitssig gehalten wird. DerSchmelzpunkt der Masse soll $60^{\circ}$ Celsins nicht tibersteigen. In der flitssigen Masse bleibt das Oljject $1 / 2$ Stande bis einen Tag.je nach seiner Grösse.

Selbst in den nenesten Publicationen lese ich, dass man allgemein das Präparat in einer Form, so gut es geht, innerhalb der flïssigen Masse in der gewünschten Stellung zu fixiren sucht und nach dem Erkalten den ganzen Block, der das Prüparat enthält, in die Zwinge des Mikrotoms einspannt. Es ist klar, dass selbst bei der grössten Sorgfalt bei solchem Vorgehen eine genaue und sichere Orientirung des Präparates nur selten gelingt, zum mindesten ausserst schwierig und umständlich ist. Es seheint mir aber grade der grösste Vorzug von Paraffin und ähnlichen Massen zu sein, dass sie nach einem Verfahren, das ich schon seit Jahren tibe, 
ein, ich möchte sagen, mathematisch genaue Orientirung des Prïparates erlauben. Ich schmelze das Präparat nicht in eine Form ein, sondern nelime es, wenn es von der Masse durebdrungen ist, herans. Bei allen Stiicken, die eine genïgende Dicke besitzen, geschieht dies mit einem vorher erhitzten Spatel; von diesem streife ich das Object mit möglichst wenig Masse anf meine Fingerspitze ab und lasse es auf dieser erkalten. Das festgewordene Sttick wird mit einigen Bewegungen vorsichtig von der Fingerspitze abgehoben und kann so in irgend einem Kastchen in Watte beliebig lange bis zum weiteren Gebrauche aufgehoben werden. Handelt es sich $\mathrm{um}$ vorzugsweise flächenhaft ansgebreitete, dünne Objecte wie z. B. Hühnerkeimscheiben der er'sten Tage, so ist ein etwas anderes Vorgehen nothwendig. Man erhitzt ein geniigend breites, ganz ebenes Blechspatel oder noch besser das eine Ende eines englischen Objectträger's, taucht os in die flissige Masse, schiebt mit der erhitzten Nadel das Prïparat darauf und hebt es beraus. Nach dem Erkalten kratzt man von der freien Seite des Spatels oder Objectträgers dic Masse ab, erwärmt dieselbe einen Augenblick an der Flamme, so dass die der andern Seite des Glases oder Spatels zunächst anhaftende Massenschicht grade flissig wird und schiebt die dadurch frei gewordenen aber noch feste Platte rasch anf die Fingerspitze ab. Von dieser nach dem rollkommenen Erkalten entfernt lassen sich solche Platten mit Keimscheiben und dergleichen vortrefflich conserriren und man erkennt an denselben durch die dunne Deckschicht von Masse hindurch nicht nur die Lage des Embryos, sondern noch eine ganze Anzahl feinerer Einzelheiten. Die ganze Manipulation ist in wenigen Augenblicken ausgefiihrt. Will man das Präparat mikrotomiren, so spanne man zuerst das Messer ein für allemal in der Stellung fest ein, die es beim Schneiden einnelimen soll.

Diese Stellung wird, wie allbekannt, für so harte Massen, wie ParaffinWachs- oder Wallrathricinusölgemische stets eine zur Fïhrungslinie des Messerschlittens annähernd senkrechte sein, während man noch für Objecte von der Resistenz gut gehärteter Leber das Messer bekanntlich so steil stellt, dass möglichst die ganze Klinge beim Schneiden ausgenützt wird. In unserem Falle kommt natïrlich nur ein kleiner Theil der KKlinge zur Geltung. Die von Dr. Long (Breslau) angegebenen Messer, dic zum Griff so gewendet sind, dass vom ganzen Messer nuj die untere Fläche des schneidenden Keils sich in der Schnittebene bewegt, dass also nicht, wio bei gewöhnlichen Messern, die untere Hälfte der Klinge äber das Prüparat hinweggedrückt wirch, sind jetzt 
wohl in der ganzen Welt bekannt und bedürfen kaum mehr einer besonderen Empfelilung.

Dann erhebe man einen in die Präparatzwinge fest eingeIrlemmten Block der Masse so boch, wie es das Instrument erlaubt und schneide auf demselben eine Ebene ab; so weiss man, dass, wenn man auf diese Ebene das Präparat aufschmilat, das Messer immer nur dieser ersten Ebene parallele Schnitte abheben wird. Es gilt also dann nur das Präparat zu der Schnittebene richtig zu orientiren. Dies geschieht bei grössseren, einfach gebauten Objecten, bei denen es anf eine absolut genaue Einstellung nicht ankommt, sehr einfach dadurch, dass man in die Mitte der Elsene auf dem Block eine Grube von entsprechender Grösse macht, in diese das harte Präparat nach dem Augenmass richtig einsetzt und dann dasselbe mit Hülfe eines gestielten, gebogenen Drahtes zuerst mit einem Tröpfchen flïssiger Masse befestigt und dann allmäblich (mit dem' erhitzten Spatel) so mit 'Tropfen der Masse einhüllt, dass das Präparat in einem Kegel der erstarrenclen Masse verschwindet. Für dünnere, kleinere und complicirte Objecte, sowie für . Fälle, bei denen eine, wie ich oben sagte, beinahe mathematisch genaue Einstellung erforderlich ist, - empfeble ich die Benutzung Ikleiner Hullsinstrumente, die man sich bei jedem Mechaniker für einen geringen Preis herstellen lassen kann. Fs sind dies zwei kleine, gleichgrosse Quadrate von planparallelem Messingblech, die an einer Seite in einem scharfen, genauen rechten Flächenwinkel mit einander verbunden sind (dieselben werden ans einem Stitck gebogen). Ich beniutze drei solcher Flïchenwinkel, bei denen die Seiten der Quadrate je 6, 8, $10 \mathrm{~m}$ messen. Auf die Aussenseite jedes Quadrats sind in gleichen Abständen drei dem einen Seitenpaar und senkrecht dariiber hinweg drei dem andern Seitenpaar paralle Linien schwarz eingravirt. Diese Flächenwinkel lassen sich sehr verschiedenartig benitzen. Das einfachste, in vielen Fällen genitgende Verfahren ist Folgendes: Man stellt den Flächenwinkel auf der Schnittebene des Blockes anf und lehnt das Prïparat so an denselben an, dass die senkreoht zul treffende Axe einer der senkrechten schwarzen Linien parallel ist und tropft es dann fest. Will das Präparat schlecht stehen, so mache man vor dem Flächenwinkel eine entsprechende Grube und dergleichen. Noch bessere Einstellung erreicht man folgendermassen: Man lege den Flächenwinkel anf die Kante eines Klötzchens und bringe das 
Präparat auf der nun horizontal vorliegenden äusseren Seite des einen Quadrates in die richtige Stellung (mit Huilfe der schwarzen Linien) und befestige es in dieser an dem Quadrat mit einem minimalen Tröpfchen der Masse. Nun stelle man den Flächenwinkel sammt dem anhängenden Präparate anf der Schnittebene des Blockes richtig ein und umgiesse das Präparat mit Ausnahme der dem Quadrat zugewendeten Seite reichlich mit Masse. Nachdem diese erkaltet ist, genitgt fast immer eine geringe Bewegung: des Flächenwinkels, $\mathrm{nm}$ die Verbindung mit dem Präparate zu lösen, das nun in der gewünschten Stellung auf dem Blocke fest bleibt and mit Leichtigkeit vollends umgossen werden kann. Wem das Erstarren zu lange dauert, braucht nur einen Pinsel mit absolutem Alkohol über dem Kegel der Masse auszudrieken und denselben anzublasen. Sollte sich einmal die Verbindung des Preiparates mit der quadratischen Platte nicht ohne Weiteres lösen lassen, so erwärme man die abgewandte Seite der letzteren mit einem in Spiritus getauchten und mit der Pincette gehaltenen, angeziindeten Wattepröpfchen, und die Lösung tritt sogleich ein. Eine Reihe pon anderen Modificationen, in denen man diese Flächenwinkel gebrauchen kann, èrgeben sich in praxi so leicht, dass ich dieselben nicht erst auseinanderzusetzen nöthig habe.

Gleichmässig dtinne Schnitte (bis $\mathrm{zu} 1 / 50 \mathrm{~m}$ und darunter) geben wohl alle modernen Mikrotome; ich kenne aus eigener Erfahirung die Instrumente von Long (Breslau) und Schanze (Leiprig) als brauchbar. Will man modelliren, so sind Schnitte von $1 / 25$ bis $1 / 50 \mathrm{~m}$ Dicke am meisten zn empfehlen; an dickeren sieht man nicht genug und verliert feinere Formübergänge; auch werden, wenn man einigermassen stark in der Fläiche vergrössert, die Wachsplatten dann so dick, dass sie sich schlecht schneiden. Nimmt man die Schnitte zu dïnn, so erschwert man sich die Arbeit dureh die Vergrösserung der Zahl der Wachsplatten unnütz und bekommt bei schwachen Vergrösserungen za diunne Platten, đie sich schlecht: giessen. Liegt ein besonderes Interesse an ganz dïnnen Schnitten vor, so schneide man z. B. $1 / 50 \mathrm{~m}$, benütze aber nur jeden zweiten Schnitt für das Modell, der dann natürlich $1 / 25 \mathrm{~m}$ dick $z$ ll rechnen ist. Man mache sich jedesmal die Flächenvergrösserung, die man ungefähr brancht, um im Modell nicht zu dỉnne Spangen und zu schmale Spalten zu erhalten, im Voraus klar und berechne darnach, wie dïn die Schnitte werden miissen, damit bei entsprechender Dicken- 
vergrösserung die Wachsplatten nicht über $2 \mathrm{~m}$ in der Dicke messen.

Fiir den Erfolg des Schneidens ist ein Hauptfactor die Masso, die man beniitzt. Dieselbe muss so beschaffen sein, dass der Schnitt einerseits nicht am Messer klebt oder sich faltet andererseits sich nicht zu einer engen Röhre zusammenrollt, sondern dass derselbe sich grade, ohme am Messer zu haften, zu einer flachen Rinne oder einfachen Röhre zusammenbiegt.

Jeder wird die leidige Erfahrung machen, dass alle ParaftinWallrath- und dergl. Gemische sehr inconstanter Natur sind, weil die Härte und Adhäsion dieser Massen in unglaublicher Weise von der Temperatur abhängt, so dass in einer solchen, die heute die vortrefflichsten Resultate gibt, wenige Tage später sich jeder Schnitt unlösbar znsammenrollt oder in Falten legt, die alle Theile heillos verschieben. Es nütut daher wenig, genaue Recepte zu geben, man kann nur im Allgemeinen sagen, dass es nützlich ist die spröden und etwas körnig krystallinischen Substanzen, wie Paraffin und Wallrath durch Zusatz von Wachs oder Ceresin mit Vaseline ${ }^{1}$ ) etwas gesehmeidiger und elastiseher zu machen.

Alle die kleinen Hitlsmittel, die F. E. Schulze ${ }^{2}$ ) fur das Aufrollen der Diinnschnitte erwähnt, habe ich selbst gefunden und durchprobirt, kann denselben sogar noch ein anderes amreihen, das sich als das brauchbarste erwiesen hat. Man wähle eine Masse, die so hart ist, dass sich ohne Weiteres jeder Schnitt einrollt; ehe man nun aber zu schneiden beginnt, umfahre man den Rand der vorliegenden Schnittebene in einiger Entfernung mit dem erhitzten hakenförmigen Encle eines gestielten Drahtes so, dass die Masse von der Wärme grade etwas afficirt wird, - das Mass lehrt Uebung sehr: bald kennen. Jetzt beginne mall rasch zu schneiden; der erste Schnitt rollt gar nicht, der zweite ein wenig, der dritte stärker, beim fïnften oder sechsten ist man genöthigt, un keinen Schnitt zu verlieren, den Handgriff zu wiederholen. Den Schnittstrecker von F. E. Scbulze besitze ich erst seit ganz lsurzer Zeit, kann das Instrumentchen jedoch als ganz vorziglich bestens empfehlen; ich befestige denselben aber nicht am Mikrotom selbst - das wäre an dem Schanze'schen Modell auch schwer zu machen, - sondern an einer eisernen Stativklammer, die daneben steht.

1) Nach M. Schulgin, Zool. Anz. VI. Jahrg. Nr. 129. p. 21.

2) Ein Schnittstrecker, Zool. Anz. 1883, Nr. 132. 
Fiir die Zweeke des Modellirens ist es selbstverständlicb unumgänglich nothwendig, dass jeder Schnitt auf dem Objecttrïger vollkommen plan ausgebreitet wird und in allen seinen Theilen, mögen dieselben auch gar keinen oder nur losen Zusammenhang haben, picht die geringste Verschiebung erleidet. Bei meinen ersten Arbeiten feuchtete ich das Messer mit absoluten Alkohol an und iubertrug in diesem auf dem Objectträger. Nach dem Verdunsten des Alc. abs. kaun man die angetrockneten Schnitte ruhig: mit Terpentin tibergiessen, ohne dass eine Verschiebung zu befürchten wäre. Nur ist eine solche leider schon während des Schneidens und Uebertragens mit Alkohol recht häufig, da die dïnnen Plättchen der Masse in Alkohol sehr brichig werden. Ich ging daher bald dazu über trocken zu schneiden, musste aber erfahren, dass zwar das trockene, feine Paraffinplättchen alle Theile des Schnittes g'tt zusammenbielt, dass aber die beim Auslösen mit Terpentin entstehenden Wirbel und Strömungen sehr leicht ganze kleinere Schnitte oder Theile von grösseren untereinanderschwemmen. Hatte man glicklich diese Klippe vermieden, so traten noch häufig, wonn man das mit Canadabalsam bestrichene Deckglas oder Glimmerblättchen auflegte, Verschiebungen ein. Die Erimnerung an moine Erfahrungen beim Feuchtschneiden brachte mich bald dazu, den Objectträger vor dem Auflegen mit absolutem Alkohol anzufeuchten, die Schnitte in denselben einzulegen, antrocknen zu lassen, fest zu scbmelzen und dann erst auszulösen; - es war mir interessant zu lesen, wie derselbe Uebelstand Gaule und Altmann ${ }^{1}$ genau auf dasselbe Gegenmittel gefithrt hat.

In neverer Zeit hat uns aber Giesbrecht ein Verfaliren geschenkt, das bei richtiger Handhabung die Lage jedes noch so losen und kleinen Schnitttheiles so vollkommen sichert, dass jedes andere Hilfsmittel entbehrlich orscheint ${ }^{2}$ ). G. gibt bekanntlich dem Objectträger einen dünnenUeberzug von alkolischer Schellacklösung, weicht denselben in der Wärme mit einem Minimum aufgepinselten Creosotes oberflächlich auf, legt dann die trockenen Paraffinschnitte

1) J. Gaule, "Das Flimmerepithel der Aricia foetida". Arch. f. Anat. u. Physiol. 1881, physiol. Abth. p. 156 Anm. u. R. Altmann, „Einige Bemerkungen über histologische Technils". Arch. f. Anat. u. Physiol. 1881, anat. Abth. p. 222 Anm.

2) ,Zur Sohneidetechnik". Zool. Anz. IV. Jahrg. Nr. 92. p. 484 und in den Mittheil. d. zool. Section zu Neapel. 1881. II. p. 184. 
auf, so dass sie an dem Sichellack thberzug festkleben, verjagt das Creosot bei $50-55^{\circ}$ und löst nachher ohne Schaden die Masse mit Strömen von Terpentin oder noeh besser Xylol aus ${ }^{1}$ ). Sollen, wie es fur das Modelliren nöthig ist, die Schnitte sich ohne Faltung ganz plan auflegen, so miissen sie sich am Besten beim Schneiden zu einer Rinne oder höchstens einfachen Röhre zusammenrollen. Diese hebt man mit der Nadel ab und legt mehrere Reihen derselben auf den Schellack auf - soviel nnter einem Deckglase oder Glimmerplättchen Platz haben, daun genuigt ein geringes Erwärmen und alle bereiten sich vollkommen plan aus und klebeu im selben Augenblicke fest.

\section{Das Modelliren.}

Das Princip der Herstellung planparaller Wachsplatten von beliebiger Dicke habe ich oben schon angedentet, die speciellere Ausfiuhrung will ich hier an einem Beispiele erläutern. Ich bentitze drei gleich grosse, rechteckige Blechgefässe mit senkrechten, etwa $2^{1 / 2} \mathrm{~cm}$ hohen Wänden; an zwei gegentiberliegenden Seiten sind Griffe angebracht. Die langen Seiten des Rechtecks messen 270 mm, die kurzen $230 \mathrm{~mm}$ im Lichten. Soll aus einer Serie von Schnitten ron $1 / 30 \mathrm{~mm}$ Dicke in 60facher Vergrösserung modellirt werden, so müssen die Wachsplatten, auf die die betreffenden Schnittheile $60 \mathrm{fach}$ vergrössert aufgezeichnet werden, ebenfalls 60 mal dicker sein, als die Schnitte, es miissen dieselben also genau $2 \mathrm{~mm}$ in der Dicke messen.

Der Flächeninhalt einer Platte, die in einem der oben beschriebenen Gefässe gegossen werden kann, beträgt 62,100 $\square \mathrm{mm}$, das Volumen einer Platte von diesem Flächeninhalt und $2 \mathrm{~mm}$ Dicke demnach 124,2 Cubiccm. - Das specifische Gewicht von gewöhnlichem gelben (rohen) Wachs beträgt $0,96-0,97$, schmilzt man das Wachs, um etwas geschmeidiger zu machen, mit einem kleinen Zusatz von Terpentin zusammen, so stellt sich das spec. Gew. auf ungefähr 0,95. Diese Zahl kann man, olne einen erbeblichen Fehler zu begehen, in allen Fällen bentitzen. Das Gewieht der fiur unsere Platten

1) Die Vorschläge zur Vervollkommnung des Giesbrecht'schen Verfahrens von Kossmann in "Zur Mikrotomtechnik. Zool. Anz. VI. Nr. 129 p. 19 u. folg. sind jedenfalls sehr beachtenswerth. 
erforderlichen Wachsmenge beträgt demnach 117,99 oder rund $118 \mathrm{gr}$. Es ist klar, dass sich das Gewicht jeder Wachsplatte voll anderer Dicke ebensoleicht berechnen Iässt. - In das Blechgefäiss giesst man zuerst kochendes Wasser bis zur Höhe von $1 \frac{1}{2} \mathrm{~cm}$, and gleich darauf das für die gewinschte Plattendicke abgewogene und dann geschmolzene Wachs.

Wenn das Wasser und das Wachs beide recht heiss sind, breitet sich das letztere ziemlich von selber aus; - Lilcken, die etwa noch bleiben, fuillt man aus, indem man mit einem breiten Objectträger oder dergl. das geschmolzene Wachs iiber dem Wasser ansstreicht. Es ist zweelsmässig, die Platte, sobald sie erstarrt, aber noch weich ist, rasch dicht an der Gefässwand za umschneiden, da bei der weiteren Abkiihlung der Gefässwand und des Wassers die Wachsplatte sonst leicht zerrissen wird. Sobald es die Festigkeit der Platte erlaubt, - das Wasser ist dann noch lan, - wird dieselbe herausgenommen und anf einer horizontalen Unterlage bis zum vollkommenen Erstarren ansgebreitet. Hat man mebrere, gleichgrosse Blechgefässe und ein par Gasflammen verschiedener Grösse zur Verfitgung, so kann ein Diener in wenigen Stunden ein halbes hundert Platten mit Leichtigkeit fertigstellen.

Am Besten und leichtesten gerathen Platten zwischen 1 und $21 / 2 \mathrm{~mm}$ Dicke, dïnnere als $0,6 \mathrm{~mm}$ habe ich wie zu machen versucht. Alle Platten werden nothwendiger Weise ein wenig' zu diünn, da sich das Waclis in Folge der Adhäsion an den Gefässwänden an den Rändern stärker anhäuft. Man könute diesen geringen Fehler noch leicht durch Rechnung eliminiren, ich. habe das aber in praxi niemals für nöthig befunden. Beim Aufeinanderleg'en der Platten bleibt zwischen ihnen ein minimaler Zwischenraum, der vielleicht diesen Fehler g'erade ausgleicht; wenigstens betrug an einem Modell, das aus 50-60 Platten zusammengesetzt war, die Differenz zwischen der aus dem idealen Plattenstärke berechneten und der gemessenen Dicke nie mehr als höchstens $1 \mathrm{~mm}$.

Früher ritzte ich die Conturen der zu modellirenden Theile mit der Nadel in die Wachsplatten ein. Es ist nun aber aus vielen Gruinden wïnschenswerth, ausser dem Bilde anf der Wachsplatte, das ja beim Ausschneiden zerstört wird, noch eine dauernde Zeichuung jedes Schnittheiles zu besitzen. Dieses Bedürfniss ver- 
anlasste wohl Swirsky, die Bilder mit Hitle der camera lucida zuerst auf Papier zu entwerfen und von diesem damn anf die Wachsplatte zu iibertragen. Folgendes Verfaluren, das ich jetzt ausschliesslich anwende, erspart den Umweg: Man breite anf der (trockenen!) Wachsplatte ein Stiuck Blaupapier (in jeder grösseren Papierhandlung zu kaufen), das eben so gross geschnitten ist, wie die erstere, so aus, dass die blane Seite dem Wachse aufliegt; anf das Blaupapier kommt ein ebenso grosser Bogen gewöhnliches Papier. Beide Bogen werden anf der Wachsplatte mit Reisszwecken befestigt. Dann zeichnet man mit einem harten, gut gespitzten Bleistift die gewitnschten Conturen auf das Papicr auf und es entsteht gleichzeitig ein congruentes Bild von blauen Linien auf der Wachsplatte. Man kaun denselben Bogen Blaupapier ungemein oft gebrauchen, ehë derselbe abgentitzt ist.

Das Ausschneiden geschieht mit einem schmalen Messer auf einer Unterlage von weichem Holz. Je nach dem Objecte wird man dabei besondere Rücksichten walten lassen. Handelt es sich um ein Object, dessen Theile im Schnitt als diunue, isolirte oder weit greschwungene Spangen erscheinen, wie z. B. bei der Modellirung eines Knorpelskelets, so lässt man beim Ausschneiden provisorische Querspangen stehen, die bis zum Aufkleben die Entfernungen sichern and nachher mit dem erwärmten Messer leicht entfernt werden. Modellirt man feine Spaltcurven, wie \%. B. Rachenhöhle und Kiemenspalten, so ist es zweckmässig, um weitere Räume zu erhalten, die Epithelien beim Aufzeichnen wegzulassen u. s. f. Gelegentlich ist man bei zu feinen Spalten, die selbst bei starker Vergrösserung noch ganz eng erscheinen, grezwungen, dieselben durchweg um ein bestimmtes Maass z. B. $1 \mathrm{~mm}$ zl weit zu zeichnen, oder man muss zu dianne Spangen um ein Bestimmtes verdicken. Solche Abweichnngen sind bei jeder. Modellirmethode unvermeidlich. Einen missglitclsten, z. B. gefalteten Schnitt, lïsst man besser ganz ats und legt dafur lieber den vorausgehenden oder nachfolgenden zweimal auf.

Besondere Vorsichtsmassregeln erfordert noch das Aufeinanderlegen der Plattenausschnitte. Es ist hier nicht möglich, alle vorkommenden Fälle zu besprechen, nur" auf einige Hauptpunkte will ich aufmerksam machen. Bei symmetrischen Figuren ist ein Abweichen nach rechts oder links natiirlich leicht zu vermeiden; auch wenn man einen Theil, der nur auf einer Seite der Symme- 
trieebene liegt, modellirt, z. B. das Labyrinthbläschen einer Seite, kann man dieselbe zweckmässig zu Hïlfe nehmen; man lässt dann regelmässig an allen Ausschnitten zwei provisorische Sparren in gleicher Höhe stehen, deren geradlinig abgeschnittene Enden genau in der Symmetrieebene liegen und passt die letzteren beim Aufeinanderlegen so ab, dass dieselben immer senkrecht auf einer und derselben Ebene stehen; man benutat als solche einen Pappdeckel oder dergleichen. Nach dem Verkleben der Ausschnitte werden die provisorischen Sparren alimählich mit dem heissen Messer entfernt. Hat man durchlaufende Gebilde in den Schnitten, deren Mittelpunkte in einer geraden Linie liegen, z. B. Wirbelkörper, so modellirt man dieselben als Hohlräume oder als Ansschnitte povisorisch mit und benutzt dieselben zar Regulirung beim Aufeinanderlegen. Auch andere durehlaufende Gebilde, wie das Ritckenmark, Blutgefässe, Chorda werden mit ausgeschnitten und belfen dann beim Aufeinanderpassen. Gegen Abweichungen in der Richtung der Symmetrieebene selbst schützt die Beridcksichtigung der Profilzeichnung des ganzen Objects, die vor dem Schneiden abgenommen wurde, und die man, wie oben erwähnt, anf die Vergrösserung des Modelles bringt mit Einzeichnung der Schnittgrenzen. Will man ganz genau sein, so kann man die Profilcontur aus dicker Pappe ausschneiden und in einen solchen Rahmen die aufeimander gelegten Schnitte einpassen. Bei unsymmetrischen Gebilden, z. B. Extremitüten, benutzt man einen senkrechten Querschnitt oder ritzt an bestimmten, dann in die Zeichnung einzutragenden Stellen ein paar Linien in die Obertläche des Präparates u. dergl. mehr.

Meist lege ich 5-6 Ausschnitte erst locker aufeinander, corrigire mit den angegebenen Hülfsmitteln die Lage und befestige sie dann aneinander, indem ich die Ränder hier und da mit dem. heissen Spatel berithre. Sobald dieselben so aneinauderhaften, dass man sie als ein Stiick aufheben tsann, bepinsele ich die vorstehenden Ränder mit Terpentin und egalisire dieselben dann mit dem heissen spatel. Die Bepinselung mit Terpentin schafft den Vortheil, dass man eine bei geringer Wärme weiche und desshalb leichter zu bearbeitende Modelloberfläche erhält.

Ob man alle Ausschnitte hintereinander weg zu einem Stiicke vollkommeu fest verkleben will, oder an bestimmten Stellen die Verklebung so oberflächlich macht, dass man das Modell wieder 
- in einzelne Stiicke zerlegen kann, wie es z. B. bei den Kiemenspaltenmodellen meiner letzten Arbeit der Fall sein musste, haingt natiùlich von Gesichtspunkten ab, welche die Beschaffenheit des jeweiligen Objects dictirt. An Stellen, die nach dem Aufeinanderpassen nachträglich wieder getrennt werden sollen, schiebt man zur Sicherung der Trennung zweckmässig ein diunnes Kartenblatt ein. Nachträglich kann man das fertige Nodell mit einem heissen Messer in jeder gewiinschten Richtung zerschneiden u. s. f. Meist arbeite ich jetzt so, dass, während ich aufzeicbne, ein paar gefällige Gehitlfen, - man kann jeden intelligenten Diener dazu rasch abrichten - die interessirenden Theile aus den fertigen Bildern ausschneiden, sodass die Herstellung des ganzen Modells relativ rasch vorwärts geht.

Man kann natürlich aus einer und derselben guten Serie alle möglichen Dinge, Hohlräume und solide Organe fuir sich modelliren. - Zum Schluss möchte ich mir nur noch erlauben, darauf hinzuweisen, dass unter anderem die Entwicklung des Gehirns und des Her\%ens sich durch meine Modelle ganz besonders gut illustriren lässt; ja man kann die Massenzunahme irgend eines Theiles des Gehirns zwischen zwei Stadien direct bestimmen, indem man aus zwei entsprechenden Modellen den Theil herausschneidet und die Wachsstticke wiegt.

Breslau, im März 1883.

Recherches sur le rein céphalique et le corps de Wolff chez les Oiseaux et les Mammifères (Extrait.)

Par lo

Dr. George Remson, Bruxelles.

Les recherches dont nous résumerons ici les résultats ont été entreprises au laboratoire de l'Institut anatomique de Strasbourg. Conduites sous la bienveillante direction de M. le Professeur Wal- 